\title{
AUTOMEDICAÇÃO E O USO IRRACIONAL DE MEDICAMENTOS: O PAPEL DO PROFISSIONAL FARMACÊUTICO NO COMBATE A ESSAS PRÁTICAS
}

\section{SELF MEDICATION AND IRRATIONAL USE OF MEDICATIONS: ROLE OF PROFESSIONAL PHARMACIST TO COMBAT THIS PRACTICE}

\author{
Wendel Simões Fernandes ${ }^{1}$ \\ Julio César Cembranelli ${ }^{2}$
}

Resumo: A automedicação e o uso irracional de medicamentos são práticas comuns entre a população brasileira, culminando em problemas secundários ocasionados por essa prática. As classes farmacológicas que estão envolvidas na automedicação são os denominados fármacos de venda livre de prescrição (MIPs), e vários fatores contribuem para a indução da prática de se automedicar, como fatores financeiros, culturais e sociais. Portanto, o profissional farmacêutico assume importante papel como orientador e agente sanitário, contribuindo para o uso racional de medicamentos e amenizando problemas relacionados ao uso inadequado de fármacos, beneficiando toda a população, que, de maneira geral, possui fácil acesso a esses profissionais.

Palavras-chave: automedicação; uso irracional; atenção farmacêutica.

Abstract: Self-medication and irrational use of drugs are common practices among the Brazilian population and culminate in secondary problems caused by this practice. The drug classes that are involved in self-medication are classified as nonprescription or over-the-counter medicine. Several factors contribute to the induction of the practice, such as financial, cultural, and social factors. Therefore, the pharmacist plays a key role as advisor and health agent, contributing to the rational use of medicines and mitigating problems related to inappropriate use of drugs. This benefits the entire population, who generally has easy access to these professionals.

Keywords: self-medication; irrational use; pharmaceutical care.

\footnotetext{
${ }^{1}$ Farmacêutico Bioquímico. Mestrando em Ciências Biológicas - Universidade do Vale do Paraíba - Univap, Brasil. Email: wen_sfernandes@hotmail.com.

${ }^{2}$ Farmacêutico Bioquímico. Pós-graduando em Farmacologia Clínica - Faculdades Oswaldo Cruz - FOC, Brasil. E-mail: julio.cembranelli@hotmail.com.
} 


\section{INTRODUÇÃO}

O profissional farmacêutico pode ser entendido como um agente de saúde de fácil acesso e encontrado na maioria das farmácias e drogarias do Brasil. A atuação desses profissionais pode contribuir muito para a população e melhorar, consideravelmente, a atual situação da saúde pública no país. A automedicação é uma conduta comum no Brasil e pode ser definida como uma forma de autocuidado, em que o indivíduo acaba consumindo medicamentos sem prescrição profissional, com o intuito de tratar e aliviar sintomas (ARRAYS, 2002). O fato de o indivíduo executar a automedicação, sem critérios técnicos e acompanhamento profissional, enquadra essa prática como uso irracional de medicamentos.

Medicamentos são de grande importância no sistema de saúde e, quando utilizados de maneira correta, cumprem seu papel no restabelecimento da homeostase e se tornam um recurso terapêutico financeiramente viável. Porém condutas que resultam no uso irracional de medicamentos podem acarretar consequências graves à saúde da população, como: reações adversas, diminuição da eficácia e dependência ao medicamento (MARIN et al., 2003). Outras consequências podem ser citadas, como: efeitos colaterais, interações medicamentosas e, até mesmo, o agravo do quadro clínico do indivíduo (SÁ et al., 2007).

De acordo com a Organização Mundial de Saúde (2008), o uso irracional de medicamentos envolve várias condutas que incluem: a utilização simultânea de muitos medicamentos sem critérios técnicos, o uso inapropriado de classes farmacológicas e prescrições médicas inadequadas. Diante da necessidade do uso criterioso de medicamentos, o farmacêutico se torna peça chave para contribuir com o uso racional.

A população, normalmente, tem fácil acesso ao profissional farmacêutico, o qual está habilitado para atuar como agente sanitário, e sua função não deve se limitar apenas à dispensação, devendo atuar de acordo com seu amplo conhecimento em favor do paciente (VIDOTTI; HOEFLER, 2006).

O Brasil, um país em pleno desenvolvimento, deseja uma assistência farmacêutica de qualidade, que garanta acesso fácil da população aos medicamentos, com qualidade e segurança. Fato esse que vem sendo trabalhado há décadas por outros países, como Estados Unidos, Canadá e Espanha (PEREIRA; FREITAS, 2008). O objetivo deste trabalho é enfatizar questões sobre a automedicação, o uso irracional de medicamentos e, ainda, demonstrar a importância do profissional farmacêutico no combate a essas práticas.

\section{MATERIAIS E MÉTODOS}

Esta revisão de literatura foi realizada com base em artigos de relevância sobre o tema proposto, permitindo a realização de uma análise qualitativa descritiva. Os artigos foram obtidos por meio de base de dados, como: Scielo, Pubmed, Bireme, Periódico Capes. Os descritores utilizados foram: Atenção farmacêutica, Automedicação, Uso irracional de medicamentos. Foram selecionados 19 artigos envolvendo o tema proposto. 
Os critérios de inclusão envolvidos foram: artigos nacionais, enquadramento do tema proposto envolvendo o período entre os anos 2000 e 2014.

\section{AUTOMEDICAÇÃO E CLASSES FARMACOLÓGICAS MAIS ENVOLVIDAS}

A automedicação é mais proeminente com os MIPs. Em estudo realizado na cidade de Salgueiro, estado do Pernambuco, envolvendo a automedicação em idosos, chegou-se à conclusão de que as classes farmacológicas mais utilizadas para automedicação foram os antipiréticos, seguidos dos analgésicos. O mesmo estudo demonstra que sintomas como febre e dores são os principais indutores da automedicação e, por se tratarem de transtornos considerados menores, e devido à precariedade da saúde pública e, muitas vezes, à dificuldade de acesso aos serviços médicos, induzem à prática de se automedicar (SÁ et al., 2007).

Em estudo realizado em Goiânia, no estado de Goiás, que, também, envolveu o uso de medicamentos por idosos, foi demonstrado que, entre os medicamentos envolvidos na prática de automedicação, os analgésicos e relaxantes musculares foram os mais citados, de um total de 461 casos de automedicação, 142 correspondem a analgésicos e relaxantes musculares. Outro dado importante, demonstrado no estudo, é a correlação entre a escolaridade e a prática da automedicação, sendo que, quanto menor a escolaridade, maior a realização dessa prática (SANTOS et al., 2013).

De acordo com os relatórios de venda de duas drogarias da cidade de Jacareí, no estado de São Paulo, referentes ao mês de agosto e setembro de 2013, entre os medicamentos de venda livre mais dispensados, destacam-se os analgésicos, sendo $63 \%$ a fração correspondente, na drogaria denominada como A; e 58\%, na drogaria denominada B.

Uma pesquisa realizada em Brasília demonstra que não só os idosos realizam a automedicação, pois os resultados encontrados revelam a utilização de sedativos e tranquilizantes, sem prescrição médica, por adolescentes (OPALEYE et al., 2013).

Um estudo envolvendo estudantes de medicina na cidade de Ribeirão Preto, estado de São Paulo, demonstrou números elevados de automedicação por esse público, e entre as classes farmacológicas mais utilizadas estão os analgésicos e antitérmicos (SILVA et al., 2012).

De acordo com um estudo realizado em Bambuí, cidade localizada no estado de Minas Gerais, os medicamentos sem prescrições mais utilizados pela população foram: analgésicos, antipiréticos, antiespasmódicos, antiácidos, antidiarreicos e vitaminas (LOYOLA FILHO et al., 2002).

Outro levantamento realizado na cidade de Santos e São Vicente, ambas no estado de São Paulo, alerta sobre a automedicação infantil. Nesse caso, os próprios pais conduzem a prática de administrar fármacos sem prescrições médicas (URBANO et al., 2010). 
Tabela 1 - Principais classes envolvidas na automedicação e respectivos

\begin{tabular}{|c|c|c|c|c|}
\hline Classes envolvidas & & $\begin{array}{l}\text { Autores } \\
\text { e país }\end{array}$ & Descrições dos estudos & Resultados \\
\hline $\begin{array}{l}\text { Analgésicos } \\
\text { antipiréticos }\end{array}$ & $\mathrm{e}$ & $\begin{array}{l}\text { Sá et al., } \\
2007 . \\
\text { Brasil }\end{array}$ & $\begin{array}{l}\text { Estudo realizado por intermédio da } \\
\text { aplicação de questionários a } 355 \\
\text { indivíduos com idade igual ou } \\
\text { superior a } 60 \text { anos, moradores da } \\
\text { cidade de Salgueiro-PE. O estudo } \\
\text { se caracteriza como de corte } \\
\text { transversal. Os dados foram } \\
\text { processados e avaliados no } \\
\text { EPIINFO } 6.04 \text {, após digitação em } \\
\text { dupla entrada e validação. }\end{array}$ & 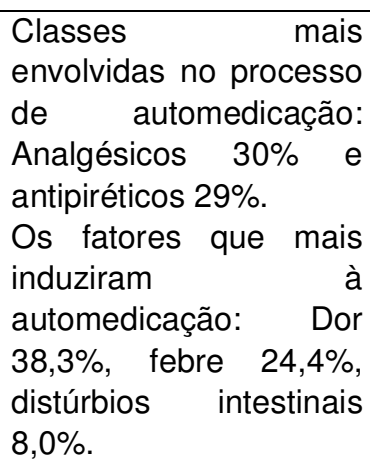 \\
\hline $\begin{array}{l}\text { Analgésicos } \\
\text { relaxante musculares }\end{array}$ & $\mathrm{s}^{\mathrm{e}}$ & $\begin{array}{l}\text { Santos et } \\
\text { al., } 2013 . \\
\text { Brasil }\end{array}$ & $\begin{array}{l}\text { Estudo de base populacional e } \\
\text { delineamento transversal, } \\
\text { envolvendo } 934 \text { idosos, da cidade } \\
\text { de Goiânia. Os dados foram } \\
\text { coletados por meio de questionário. } \\
\text { Os testes utilizados foram Qui- } \\
\text { quadrado e exato de Fisher. }\end{array}$ & $\begin{array}{l}\text { Os fármacos mais } \\
\text { envolvidos no processo } \\
\text { de automedicação por } \\
\text { idosos foram os } \\
\text { analgésicos }(30,8 \%) \text {. }\end{array}$ \\
\hline $\begin{array}{l}\text { Anal } \\
\text { antip }\end{array}$ & $\mathrm{e}$ & $\begin{array}{l}\text { Silva et } \\
\text { al., } 2012 . \\
\text { Brasil }\end{array}$ & $\begin{array}{l}\text { Estudo transversal quantitativo, } \\
\text { realizado por meio da aplicação de } \\
\text { questionários a estudantes de } \\
\text { medicina da Universidade de } \\
\text { Medicina, de Ribeirão Preto, com o } \\
\text { objetivo de se determinar a } \\
\text { automedicação no público em } \\
\text { questão. Para a análise estatística, } \\
\text { foi utilizado o teste de correlação, de } \\
\text { Pearson. }\end{array}$ & $\begin{array}{l}\text { Medicamentos mais } \\
\text { envolvidos no processo } \\
\text { de automedicação: } \\
\text { analgésicos } \\
\text { antipiréticos } 90,2 \% \text {. }\end{array}$ \\
\hline $\begin{array}{l}\text { Analgésico, } \\
\text { antipiréticos, } \\
\text { antiespasmódicos, } \\
\text { antiácidos, } \\
\text { antidiarreicos, } \\
\text { vitaminas }\end{array}$ & & $\begin{array}{l}\text { Loyola } \\
\text { Filho et } \\
\text { al., } 2002 . \\
\text { Brasil }\end{array}$ & $\begin{array}{l}\text { Estudo realizado por meio de } \\
\text { aplicação de questionários a } 1221 \\
\text { moradores da cidade de Bambuí- } \\
\text { MG, com o objetivo de se } \\
\text { determinar fatores associados à } \\
\text { automedicação. Para a análise } \\
\text { estatística, foram utilizados os } \\
\text { testes de Qui-quadradro, de } \\
\text { Pearson e odds ratio. }\end{array}$ & $\begin{array}{lr}\text { Analgésicos } & \mathrm{e} \\
\text { antipiréticos } & 47,6 \% \text {, } \\
\text { antiespasmódicos, } \\
\text { antiácidos } \\
\begin{array}{l}\text { antidiarreicos } \\
\text { vitaminas } 4,7 \% \text {. }\end{array}\end{array}$ \\
\hline
\end{tabular}

\subsection{Os principais fatores que induzem a automedicação}

Vários podem ser os fatores que induzem a prática de automedicação no Brasil. De acordo com Urbano e colaboradores (2010), muitos casos de automedicação são induzidos com base em prescrições antigas e, também, por indicações de terceiros.

Outro fator atribuído à prática da automedicação é a presença de dores, o que leva o paciente a buscar uma solução rápida, buscando assim na automedicação a resolução do sintoma (SÁ et al., 2007). 
Um ponto interessante relatado por um estudo, como sendo indutor da prática de automedicação, é a padronização atual de prescrições, o que faz com que a população adote critérios próprios para solucionar problemas de saúde de menor gravidade. O mesmo estudo aponta ainda outros fatores envolvidos na automedicação, entre eles, poder aquisitivo, questões culturais e acesso à informação, além da escolaridade (BORTOLON et al., 2007; SCHIMD et al., 2010; SANTOS et al., 2013).

No Brasil, onde o acesso à saúde é difícil e grande parte da população não apresenta condições financeiras para custear um plano particular de saúde, o ato de se automedicar se torna comum, tornando-se uma questão até mesmo cultural (NASCIMENTO et al., 2005).

Outros aspectos podem ser apontados como indutores da automedicação no Brasil, como o não cumprimento da obrigatoriedade da prescrição no ato da dispensação, favorecendo a automedicação de fármacos que, legalmente, necessitam da apresentação da prescrição para a venda (SOUZA et al., 2008).

\subsection{O papel do farmacêutico no combate à automedicação e ao uso irracional de medicamentos}

O profissional farmacêutico deve ser encarado como um agente da saúde, responsável por ofertar orientações técnicas de confiança sobre medicamentos, baseado no amplo conhecimento dessa classe de profissionais (SERAFIM et al., 2007). As diretrizes curriculares nacionais para os cursos de Farmácia salientam o múltiplo conhecimento que deve ser adquirido pelo farmacêutico, não envolvendo somente o de caráter técnico-científico, mas também habilidades sobre outras áreas, como ética e humanização (POSSAMAl; DECOREGGIO, 2008).

A farmácia atualmente é uma porta de acesso primário à saúde em nosso país, sendo o farmacêutico procurado, muitas vezes, antes de um serviço hospitalar. Dessa maneira, o farmacêutico, dentro de suas habilitações e possibilidades, deve estar preparado para atuar de maneira adequada, executando a atenção farmacêutica sempre a favor do paciente (GALATO et al., 2008).

A atenção farmacêutica é a ferramenta utilizada pelo profissional farmacêutico, com o objetivo de promover o uso racional de medicamentos e conscientizar a população sobre a importância dessa prática, justificando a necessidade da presença desse profissional em todas as farmácias e drogarias do país (SOUSA et al., 2008).

Uma questão muito interessante contra a prática da automedicação e a favor do uso racional de medicamentos é a legalização da prescrição farmacêutica, por intermédio da resolução 586 de 29 de agosto de 2013, que estabelece a prescrição farmacêutica sobre medicamentos de venda livre.

De acordo com Bortolon e colaboradores (2007), os MIPs só fazem sentido se sua dispensação ocorrer sob orientação profissional, pois, apesar de serem fármacos que 
apresentam relativa segurança, podem acarretar reações alérgicas, efeitos adversos e colaterais e interações medicamentosas de relevância.

Dessa forma, a prescrição farmacêutica se torna de grande utilidade, uma vez que transforma a automedicação em uma indicação farmacêutica realizada com critérios, favorecendo o uso racional de medicamentos. Vale ressaltar que os fármacos de venda livre se enquadram entre as classes mais utilizadas para automedicação.

Em determinada pesquisa, foi demonstrado que boa parte da população não possui bom nível de informação sobre medicamentos, apresentando dúvidas sobre a maneira correta de utilizá-los e, até mesmo, sobre a indicação terapêutica do fármaco. Outro resultado obtido na mesma pesquisa demonstra que, após a dispensação dos fármacos, o nível de conhecimento sobre o medicamento é melhor por parte do consumidor, o que pode torná-lo mais criterioso sobre a automedicação, justificando, dessa maneira, a importância da atuação farmacêutica diante da dispensação (OENNING et al., 2011).

A atenção farmacêutica é uma estratégia para o uso racional de medicamentos, pois por intermédio dela o paciente recebe várias informações e orientações com o objetivo de maximizar a farmacoterapia.

Orientar o paciente sobre o uso de medicamentos não é atribuição exclusiva do farmacêutico, mas seu conhecimento amplo sobre fármacos e sua atuação ativa na dispensação, conferem a esse profissional oportunidade única para atuar a favor do uso racional (POSSAMAI; DECOREGGIO, 2008), reforçando o papel do farmacêutico no combate a essas práticas.

A atuação do farmacêutico deve ser embasada levando em conta aspectos fisiológicos e patológicos dos pacientes, atribuindo, assim, condições para se realizar a prescrição farmacêutica (BORTOLON et al., 2007). O que deve ser bem estruturado nesse processo é o limite de atuação, portanto, em situações que se julgar necessário, o farmacêutico sempre deve orientar o paciente a buscar orientação médica adequada, e conscientizar o paciente sobre essa necessidade.

A profissão farmacêutica, que já passou por várias dificuldades diante da sua trajetória, encontra-se em um momento único, perante a oportunidade de atuar por meio da atenção farmacêutica, somando-se a isso a carência da população ao acesso a serviços de saúde de qualidade (VIERA, 2007).

Dessa maneira, o profissional farmacêutico deve assumir a responsabilidade de promotor da saúde e contribuir a favor do uso racional de medicamento, favorecendo a população brasileira e desafogando a saúde pública do país.

\section{CONCLUSÃO}

Analisando os dados encontrados sobre automedicação e o uso irracional de 
medicamentos e as classes citadas nas pesquisas, podemos inferir que a prescrição farmacêutica apresenta-se como ferramenta de segurança, garantindo aos pacientes o uso correto de medicamentos, sendo o principal beneficiado da atuação farmacêutica a população brasileira.

\section{REFERÊNCIAS}

ARRAYS, P. S. D. O uso irracional de medicamentos e a farmacovigilância no Brasil. Cad. Saúde Pública, Rio de Janeiro, v. 18, p. 1478-1479, set./out. 2002.

BORTOLON, P. C.; KARNIKOWSKI, M. G. O.; ASSIS, M. Automedicação versus indicação farmacêutica: o profissional de farmácia na atenção primária a saúde do idoso. Rev. APS, Juiz de Fora, v. 10, n. 2, p. 200-209, jul./dez. 2007.

GALATO, D. et al. A dispensação de medicamentos: uma reflexão sobre o processo para prevenção, identificação e resolução de problemas relacionados a farmacoterapia. Rev. bras. ciênc. farm., São Paulo, v. 44, p. 465-475, jul./set. 2008.

LOYOLA FILHO, A. I. et al. Prevalência e fatores associados à automedicação: resultados do projeto Bambuí. Rev. Saúde Pública, São Paulo, v. 36, n. 1, p. 55-62, fev. 2002.

MARIN, N. (org.). et al. Assistência farmacêutica para gerentes municipais. Rio de Janeiro: OPAS; OMS, 2003. p. 287-334.

OENNING, D.; OLIVEIRA, B. V.; BLATT, C. R. Conhecimento dos pacientes sobre os medicamentos prescritos após consulta médica e dispensação. Ciênc. saúde coletiva, Rio de Janeiro, v. 16, p. 3277-3283. 2011.

OPALEYE, E. M. et al. Nonprescribed use of tranquilizers or sedatives by adolescents: a Brazilian national survey. BMC public health, London, v.13, p. 499, 2013.

ORGANIZAÇÃO MUNDIAL DA SAÚDE. Selection and rational use of medicines. Disponível em: <http://www.who.int/medicines/areas/rational use/en/index.html>. Acesso em: 12 mar. 2014.

PEREIRA, L. R. L.; FREITAS, O. A evolução da atenção farmacêutica e a perspectiva para o Brasil. Rev. Bras. Cienc. Farm., São Paulo, v. 44, p. 601-612, out./dez. 2008.

POSSAMAI, F. P.; DACOREGGIO, M. S. A habilidade de comunicação com o paciente no processo de atenção farmacêutica. Trab. educ. saúde, Rio de Janeiro, v. 5, p. 473-490, 2008.

SÁ, M. B.; BARROS, J. A. C.; SÁ, M. P. B. O. Automedicação em idosos na cidade de Salgueiro - PE. Rev. bras. epidemiol., São Paulo, v. 10, n. 1, p. 75-85, 2007.

SANTOS, T. R. A. et al. Consumo de medicamentos por idosos, Goiânia, Brasil. Rev. Saúde Pública, São Paulo, v. 47, p. 94-103, 2013.

SCHMID, B.; BERNAL, R.; SILVA, N. N. Automedicação em adultos de baixa renda no 
município de São Paulo. Rev. Saúde Pública, São Paulo, v. 44, p. 1039-45, 2010.

SERAFIM, E. O. P. et al. Qualidade dos medicamentos contendo dipirona encontrados nas residências de Araraquara e sua relação com a atenção farmacêutica. Rev. Bras. Cienc. Farm., São Paulo, v. 43, p. 127-135, jan./mar. 2007.

SILVA, R. C. G. et al. Automedicação em acadêmicos de medicina. Medicina, Ribeirão Preto, v. 45 , p. $5-11,2012$.

SOUSA, H. W. O.; SILVA, J. L.; NETO, M. S. A importância do profissional farmacêutico no combate a automedicação no Brasil. Rev. eletrônica farm., Goiânia, v. 8, p. 67-72, 2008.

URBANO, A. Z. R. et al. Automedicação infantil: O uso indiscriminado de medicamentos nas cidades de Santos e de São Vicente. Revista Ceciliana, Santos, v. 2, n. 2, p. 6-8. 2010.

VIDOTTI, C. C. F.; HOEFLER, R. Apoio a transformação do exercício profissional do farmacêutico na farmácia comunitária. Boletim Farmacoterapêutica. Brasília, v. 11, n. 1, p. 1-5, jan./fev. 2006.

VIEIRA, F. S. Possibilidades de contribuição do farmacêutico para a promoção da saúde. Ciênc. saúde coletiva, Rio de Janeiro, v. 12, n. 1, p. 213-220, mar. 2007. 